\title{
Regression-Tensor Modeling of Multivariate Optimization of Process for Applying Metal Coatings
}

\author{
Vyacheslav Anatolievich Rusanov', Sergey Viktorovich Agafonov², \\ Sergey Nikolayevich Dumnov³, Aleksey Vasilyevich Daneev ${ }^{3}$, Sergey Vasilyevich Lyamin ${ }^{3}$ \\ ${ }^{1}$ Institute for System Dynamics and Control Theory of Siberian Branch of Russian Academy of Sciences (ISDCT \\ SB RAS), Irkutsk, Russia \\ ${ }^{2}$ Irkutsk State Agricultural Academy (ISAA), Irkutsk, Russia \\ ${ }^{3}$ Irkutsk State Transport University (ISTU), Irkutsk, Russia \\ Email: v.rusanov@mail.ru, daneev@mail.ru
}

Received 7 October 2014; revised 7 November 2014; accepted 21 November 2014

Copyright (C) 2014 by authors and Scientific Research Publishing Inc.

This work is licensed under the Creative Commons Attribution International License (CC BY).

http://creativecommons.org/licenses/by/4.0/

c) (i) Open Access

\section{Abstract}

A universal regression-tensor approach is developed in the mathematical modeling of optimal parameters of chemical-technological process of complex mechanical products. The testing of developed algorithms was performed on the example of multi-factorial process of low-temperature sulfur-chromium plating of precision mechanical parts.

\section{Keywords}

Covariant Tensors of Final Valence, Identification of Nonlinear Vector Regression, Optimization of Metal Coatings

\section{Introduction}

Originally regression analysis acquired theoretical and applied interest in problems of optimizing the parameters of linear stationary systems (type "black box"). In most cases, the studies were limited with analysis of finitedimensional systems [1] [2]; as a rule, the problem of identification of regression was formulated in terms of computing quadratic-optimal estimation of model parameters using the method of least squares with following application of constructing pseudo-inverse matrix ([3] p. 186).

In this article regression analysis differs from the traditional presentation [1] [2] because one of the main goals was to present more clearly geometrical (in the language of constructions [4] [5]) device of multivariate 
nonlinear regression modeling [6] (with simultaneous reduction of dimension of used pseudo-matrix). According to this applied interpretation of theoretical results of the article [6] is studied in details below; it is interesting in connection with other applications of method of least squares-interpolation where generalizations of algorithmic solutions proposed in [6] can manifest.

The approbation of theoretical apparatus of nonlinear vector regression in the article stands the problem of optimization (as a way of technological calculation) of characteristics of multi-factorial chemical-technological process. Payment of optimization of process of low-temperature sulfur-chromium plating of precised mechanical products is taken as example.

\section{The Statement of the Problem of Modeling of Optimal Chemical-Technological Process}

Let $R$-field of real numbers, $R^{n}$-n-dimensional vector space over $R$ with Euclidian norm $\|\cdot\| R^{n}$, $\operatorname{col}\left(y_{1}, \cdots, y_{n}\right) \in R^{n}$ — column vector with elements $y_{1}, \cdots, y_{n} \in R$ and let $M_{n, m}(R)$-space of all $n \times m$-matrices with elements from $R$ and Frobenius matrix norm $\|D\|_{F}=\left(\Sigma d_{i j}^{2}\right)^{1 / 2}, D=\left[d_{i j}\right]$. Further through $T_{m}^{k}$ we denote the space of all covariant tensors $k$-th valence (real multi-linear forms $f^{k, m}: R_{1}^{m} \times \cdots \times R_{k}^{m} \rightarrow R$ ) with tensor norm $\left\|f^{k, m}\right\|_{T}=\left(\Sigma t_{i \cdots j}^{2}\right)^{1 / 2}$, where $t_{i \cdots j}$-coefficients ([4] p. 61) of a tensor $f^{k, m}$, the values of which are relative to the standard algebraic basis ([7] p. 15) in the Euclidean space $R^{m}$.

Let $v \in R^{m}$ - vector of varied physicochemical predictors ([2] p. 38) of regression of chemical-technological process with fixed origin in $\omega \in R^{m}$ (reference mode), $w(\omega+v) \in R^{n}$ - vector of qualitative indexes of chemical-technological process. In this setting we select for consideration a nonlinear system of type "input-output" described by vector-tensor $k$-valent equation of multiple regression

$$
w(\omega+v)=c+A v+\operatorname{col}\left(\sum_{j=2, \cdots, k} f_{1}^{j, m}(v, \cdots, v), \cdots, \sum_{j=2, \cdots, k} f_{k}^{j, m}(v, \cdots, v)\right)+\varepsilon(\omega, v)
$$

where $v \in R^{m}, \quad c \in R^{n}, A \in M_{n, m}(R), \quad f_{i}^{j, m} \in T_{m}^{j}$, vector-function $\varepsilon(\omega,):. R^{m} \rightarrow R^{n}$ satisfies the condition $\|\varepsilon(\omega, v)\| R_{n}=o\left(\left(v_{1}^{2}+\cdots+v_{2}^{2}\right)^{k / 2}\right), \quad v=\operatorname{col}\left(v_{1}, \cdots, v_{m}\right)$.

The statement of the problem of optimization of chemical-technological process consists of three steps:

(i) for a fixed index $k$ given $\omega \in R^{m}$, vector-function of chemical-technological process $w: \Omega \rightarrow R^{n}$, where $\Omega \subset R^{m}$-open area with a point $\omega$, to determine the conditions under which the mapping $w(\cdot)$ satisfies the system (1) with some $c, A, f_{i}^{j, m}, 1 \leq i \leq n, 1 \leq j \leq k$;

(ii) to build a posteriori estimates $c, A, f_{i}^{j, m}, 1 \leq i \leq n, 1 \leq j \leq k$ from solution of two-criteria problem of parametric regression model (1):

$$
\left\{\begin{array}{l}
\min \left(\sum_{1 \leq l \leq q}\left(\left\|w_{(l)}-c-A v_{(l)}-\operatorname{col}\left(\sum_{j=2, \cdots, k} f_{i}^{j, m}\left(v_{(l)}, \cdots, v_{(l)}\right), \cdots, \sum_{j=2, \cdots, k} f_{n}^{j, m}\left(v_{(l)}, \cdots, v_{(l)}\right)\right)\right\| R^{n}\right)^{2}\right)^{1 / 2} \\
\min \left(\|c\|_{R^{n}}^{2}+\|A\|_{F}^{2}+\sum_{i=1, \cdots, n} \sum_{j=2, \cdots, k}\left\|f_{n}^{j, m}\right\|_{T}^{2}\right)^{1 / 2}
\end{array}\right.
$$

here $w_{(l)} \in R^{n}, v_{(l)} \in R^{m}, 1 \leq l \leq q$ are vectors of experimental data ( $w_{(l)}$ - "reaction" on "variation” $v_{(l)}$ with respect to "point" of reference mode of chemical-technological process $\omega \in R^{m}$ ), $q$-total number of experiments of chemical-technological process, thus we don't impose restrictions on the magnitude $q$; this number should be "sufficient" by solving the problem of parametric identification tensor structure of Equation (1) was uniquely defined (see also below Remark 2);

(iii) for $\varepsilon(\omega, v)=0$ and fixed $\omega \in R^{m}, k$ to determine vector of "input" variables of chemical-technological process $v^{*} \in R^{m}$ from solution of problem " $v$-optimization" (construction of weighted averaged optimal characteristics of "output" variables of chemical-technological process): 


$$
\max \left\{F(v): v \in R^{m}\right\}, F(v)=\sum_{i=1, \cdots, n} r_{i} w_{i}(\omega+v)
$$

where $r_{i}$-specified weighted coefficients of weighted averaged assessment of chemical-technological process and variables of vector-function $\operatorname{col}\left(w_{1}(\omega+v), \cdots, w_{n}(\omega+v)\right)=w(\omega+v) \in R^{n}$ have analytical representation in force of identified model (1), i.e. according to (ii).

\section{Existence of a Model of Multivariate Regression of Chemical-Technological Process}

In this section we will examine the analytic properties of nonlinear vector regressions of many variables that look like behavior of holomorphic functions (problem (i) from paragraph 1). In connection with this the presentation will be based on the concept of the Frechet derivative ([5] p. 48). Last poses the problem of determining the remaining concepts in particular the higher order differentials through the design of these derivatives; it is known ([5] p. 490), that Frechet $k$-derivatives can be (and most comfortable) interpreted as mathematical constructions with multi-linear ( $k$-linear) structure that reflects the following:

Proposition 1. Let $\Omega$-open area in $R^{m}, w(\cdot): \Omega \rightarrow R^{n}$ and $\omega$-point from $\Omega$. If there is a Frechet derivative of order $k$, then the Frechet differential of $k$-th order $d^{k} w$ for $w(\cdot)$ in point $\omega \in \Omega$ of increment $v \in R^{m}$ has a representation

$$
d^{k} w=w^{(k)}(\omega)(v, \cdots, v)=\operatorname{col}\left(f_{1}^{k, m}(v, \cdots, v), \cdots, f_{n}^{k, m}(v, \cdots, v)\right), f_{i}^{k, m} \in T_{m}^{k}, i=1, \cdots, n
$$

Proof. Each derivative $w^{(k)}(\omega)$ can be associated with an element of space of $k$-linear mappings from $R_{1}^{m} \times \cdots \times R_{k}^{m}$ in $R^{n}$ ([5] p. 490). On the other hand, covalent tensor of $k$-th valency is ([4] p. 58) multi-linear functional on $R_{1}^{m} \times \cdots \times R_{k}^{m}$ that makes fair (4). $\square$

Before we take a further step we note that formulation of Proposition 1 essentially imposes on the map $w(\cdot)$ one additional requirement, namely, position of analytical representation of vector-function $w(\cdot)$. In the case of a posteriori modeling $w(\cdot)$ this requirement is not feasible, so above we are limited with the analysis according to the problem (i) less realistic, but more logically verified task of analysis of the properties of mapping $w(\cdot)$.

In next assertion we establish an important property which a vector-function $w(\cdot)$ should have clarifying: when the mapping $w(\cdot)$ satisfies at least under some reasonable assumptions on it one of those special laws from which a concept of tensor regression (1) happened as a natural product of a continuous process of consolidation, abstraction and generalization.

Proposition 2. Let $\Omega$-open domain in $R^{m}$, w(.)-mapping of a set $\Omega$ in $R^{n}$ and $\omega$-some point from $\Omega$. If there is a Frechet derivative $w^{(k)}(\omega)$, which is a uniformly continuous function of $\omega$ in $\Omega$, then vector-mapping $w(\cdot): \Omega \rightarrow R^{n}$ satisfies (1) with some tensors $f_{i}^{j, m} \in T_{m}^{j}, 1 \leq i \leq n, 1 \leq j \leq k$, the vector $c=w(\omega) \in R^{n}$ and matrix $A=w^{(1)}(\omega) \in M_{n, m}(R)$.

Proof. By theorem 2 ([5] p. 491) uniform continuity of the strong derivative $w^{(k)}(\cdot)$ of mapping $w(\cdot): \Omega \rightarrow R^{n}$ means that vector difference $w(\omega+v)-w(\omega)$ can be represented as the sum of a finite vector number expressed by the Formula (21) ([5] p. 491) (similar to the canonical formula of Taylor for power series of realvalued function):

$$
w(\omega+v)=w(\omega)+w^{(1)}(\omega) v+w^{(2)}(\omega)(v, v) / 2+w^{(k)}(\omega)(v, \cdots, v) / k !+\varepsilon(\omega, v)
$$

where vector-function $\varepsilon(\omega, \cdot)$ of class $\|\varepsilon(\omega, v)\| R^{n}=0\left(\left(v_{1}^{2}+\cdots+v_{m}^{2}\right)^{k / 2}\right), \quad v=\operatorname{col}\left(v_{1}, \cdots, v_{m}\right)$.

Thus, the compilation of this proposal with Formula (4) leads to

$$
w(\omega+v)=c+A v+\operatorname{col}\left(\sum_{j=2, \cdots, k} f_{1}^{j, m}(v, \cdots, v), \cdots, \sum_{j=2, \cdots, k} f_{n}^{j, m}(v, \ldots, v)\right)+\varepsilon(\omega, v)
$$

where $c \in R^{n}, A \in M_{n, m}(R), \quad f_{i}^{j, m} \in T_{m}^{j}, j=2, \cdots, k$.

Remark 1. Everywhere further we believe a priori that the simulated chemical-technological process satisfies the Proposition 2 for some index $k \geq 2$. 
The Proposition 2 provides the most direct way of interpreting the concept of model complexity because it shows a direct link between the approximate model and the way the model should be evaluated from experimental data which in the strict sense refuse it; when we set the maximum allowable inconsistency $\varepsilon(\omega, v)$ and in the corresponding class of regression models we seek the least complicated object (with a minimal tensor valency $k$ ). In this part Proposition 2 essentially formulates qualitative fact for the existence of regression (1) if we don't impose overly strict requirements on the analytical structure of vector-function $w(\cdot)$.

\section{Identification of Quadratic-Vector Regression Model of Chemical-Technological Process}

Let's start with specification of tensor construction of Equation (1); this specification has a special character but its use in potential allows not to attract complex computational algorithms for calculating an optimal vector of variables of chemical-technological process. We consider (including Remark 1) the case $k=2$. We also agree that the coordinates $t_{i j}$ of each tensor $f_{i}^{2, m} \in T_{m}^{2}(1 \leq i \leq n)$ a priori satisfies the condition $t_{i j}=0, i \geq j$. In this formulation the Equation (1) takes the form:

$$
w(\omega+v)=c+A v+\operatorname{col}\left(v^{\mathrm{T}} B_{i} v, \cdots, v^{\mathrm{T}} B_{n} v\right)+\varepsilon(\omega, v)
$$

where $B_{i} \in M_{m, m}(R), i=1, \cdots, n$ wherein each $B_{i}$-an upper triangular matrix ([7] p. 38); here and further the symbol “T”- operation of vector-matrix transposition.

By Proposition 2 and Theorem 12 ([8] p. 189) we have an obvious interpretations:

$$
\begin{gathered}
c=w(\omega) \in R^{n} \\
A=w^{(1)}(\omega) \in M_{n, m}(R), \operatorname{col}\left((.)^{\mathrm{T}}\left(B_{1}+B_{1}^{\mathrm{T}}\right)(.) \cdots(.)^{\mathrm{T}}\left(B_{n}+B_{n}^{\mathrm{T}}\right)(.)\right)=w^{(2)}(\omega)
\end{gathered}
$$

here $w^{(k)}(\omega)-k$-Frechet derivative (in point $\omega$ ) of vector-function $w(\cdot): \Omega \rightarrow R^{n}$.

We will associate methodologically the parametric identification in multi-criteria vector-matrix-tensor formulation (2) for multiple stationary nonlinear model of type "black box" in class of regressions (5) with the concept of normal pseudo-solution for a finite-dimensional system of linear algebraic equations.

As usual ([7] p. 501) normal pseudo-solution of system of algebraic equations $D x=d, D \in M_{q, p}(R)$, $d \in R^{q}$, is the vector $x \in R^{p}$, which has the smallest norm $\|x\| R^{n}$ among all vectors yielding minimum $\|D x-d\| R^{n}$. Next we denote through $E_{q}$ —unit $q \times q$-matrix and let $D \in M_{q, p}(R)$, while through $D^{+}$we denote the generalized inverse (pseudo-inverse) matrix of Moore-Penrose ([7] p. 500) of matrix $D$; asymptotic construction of pseudo-inverse matrix has the following analytical form of

$$
D^{+}=\lim \left\{D^{\mathrm{T}}\left(D D^{\mathrm{T}}+\tau E_{q}\right): \tau \rightarrow 0 \in R\right\}
$$

Then (see Formula (50), [8] p. 35) vector $D^{+} d$-normal pseudo-solution of system of linear algebraic equations $D x=d$; we agree further to denote by symbol “+” the operation of the corresponding matrix.

We assume that during the operation of chemical-technological process there were conducted $q$-experiments of type "input-output”. For parameters of quadratic regression system (5) and $q$-data (general sample) of conducted experiments we denote through $\hat{u}_{(l)} \in R^{m(m+3) / 2}, 1 \leq l \leq q$ vector of "input" variables having the upper triangular structure of each matrix $B_{i} \in M_{m, m}(R), 1 \leq i \leq n$, the following coordinate representation:

$$
\begin{gathered}
\hat{u}_{(l)}=\operatorname{col}\left(v_{1(l)}, \cdots, v_{m(l)}, v_{1(l)} v_{1(l)}, \cdots, v_{r(l)} v_{s(l)}, \cdots, v_{m(l)} v_{m(l)}\right) \in R^{m(m+3) / 2}, 1 \leq r \leq s \leq m \\
\operatorname{col}\left(v_{1(l)}, \cdots, v_{m(l)}\right)=v_{(l)} \in R^{m}, 1 \leq l \leq q
\end{gathered}
$$

We call complete matrix of experimental data of input variables of chemical-technological process (6) $q \times$ $m(m+3) / 2$-matrix of type

$$
U=\left[\hat{u}_{(1)}, \cdots, \hat{u}_{(l)}, \cdots, \hat{u}_{(q)}\right]^{\mathrm{T}} \in M_{q, m(m+3) / 2}(R)
$$


respectively vector

$$
\hat{w}_{i}=\operatorname{col}\left(w_{i(1)}-w_{i}(\omega), \cdots, w_{i(l)}-w_{i}(\omega), \cdots, w_{i(q)}-w_{i}(\omega)\right) \in R^{q}
$$

we will call the complete vector of experimental data of $i$-th output variable.

Further considering that in system (5) each matrix $B_{i}$ is upper triangular the structure of $i$-th equation $(i=1, \cdots, n)$ of this system will take the form:

$$
w_{i}(\omega+v)=c_{i}+\sum_{1 \leq j \leq m} a_{i j} v_{j}+\sum_{1 \leq r \leq s \leq m} b_{i r s} v_{r} v_{s}+\varepsilon_{i}(\omega, v)
$$

It is clear that in view of algebraic structure of Equation (7) the problem of parametric identification (2) should be solved on some basis of $q$-experiments with respect to the next group of vectors (of dimension $m(m+3) / 2)$ :

$$
\begin{gathered}
z_{1}=\operatorname{col}\left(a_{11}, \cdots, a_{1 m}, b_{111}, \cdots, b_{1 r s}, \cdots, b_{1 m m}\right) \in R^{m(m+3) / 2}, 1 \leq r \leq s \leq m \\
\vdots \\
z_{i}=\operatorname{col}\left(a_{i 1}, \cdots, a_{i m}, b_{i 11}, \cdots, b_{i r s}, \cdots, b_{i m m}\right) \in R^{m(m+3) / 2}, 1 \leq r \leq s \leq m \\
\vdots \\
z_{n}=\operatorname{col}\left(a_{n 1}, \cdots, a_{n m}, b_{n 11}, \cdots, b_{n r s}, \cdots, b_{n m m}\right) \in R^{m(m+3) / 2}, 1 \leq r \leq s \leq m
\end{gathered}
$$

it is obvious that this group of vectors completely determines (sets) elements of matrix $A=w^{(1)}(\omega) \in M_{n, m}(R)$ and matrices $B_{i} \in M_{m, m}(R), \quad i=1, \cdots, n$ in the structure $w(2)(\omega)$ of regression equations of the system (5); it is clear that the vector $c$ sets the reference mode:

$$
c=w(\omega) \in R^{n}
$$

Now we can give the solution to the problem of parametric identification of model of bilinear-tensor regression of chemical-technological process only by a posteriori information on the basis of preliminary passage of $q$ experiments.

Proposition 3. The problem of identification (2) in terms of parameters (8) of regression model (5) has a solution:

$$
z_{i}^{*}=U^{+} \hat{w}_{i}, 1 \leq i \leq n
$$

where $U$-full matrix of experimental data of input actions (6), $\hat{w}_{i}$-full vector of experimental data of $i$-th output variable of chemical-technological process $(i=1, \cdots, n)$, induced with variables (6).

Proof. Below we will give a sketch of the proof. Following the standard arguments regression (5) for each l-th experiment according to the relations (6), (7) takes the following compact form:

$$
w_{i(l)}-w_{i}(\omega)=\breve{u}_{(l)} Z_{i}+\varepsilon_{i(l)}, i=1, \cdots, n
$$

Thus, if we reformulate according to the last system, optimization problem of parametric identification (2) applied to the equations of regression in tensor structure (5), then we arrive to the following multi-criteria formulation with respect to vectors $z_{i}, i=1, \cdots, n$ :

$$
\begin{gathered}
\left\{\begin{array}{c}
\min \left\|\hat{w}_{1}-U z_{1}\right\| R^{q}, \\
\min \left\|z_{1}\right\| R^{m(m+3) / 2},
\end{array}\right. \\
\vdots \\
\left\{\begin{array}{c}
\min \left\|\hat{w}_{l}-U z_{i}\right\| R^{q}, \\
\min \left\|z_{i}\right\| R^{m(m+3) / 2}, \\
\vdots
\end{array}\right. \\
\left\{\begin{array}{l}
\min \left\|\hat{w}_{n}-U z_{n}\right\| R^{q}, \\
\min \left\|z_{n}\right\| R^{m(m+3) / 2}
\end{array}\right.
\end{gathered}
$$


It is not difficult to establish that this multi-criteria formulation has (according to Formula (50), [9] p. 35) only one normal pseudo-solution $U^{+} \hat{w}_{i}, 1 \leq i \leq n$ with respect to vectors $z_{i}, 1 \leq i \leq n$.

Corollary 1 ([10] p. 263). Let $z_{i}^{*}=U^{*} \hat{w}_{i}, 1 \leq i \leq n$, then each vector $z \in R^{m(m+3) / 2}$ of parameters of regression model (5) which characterizes the behavior of chemical-technological process such that $z \neq z_{i}^{*}$ satisfies one of the following two conditions:

(*) $\min \left\|\hat{w}_{i}-U z\right\| R^{q}>\min \left\|\hat{w}_{i}-U z_{i}^{*}\right\| R^{q}$, or otherwise we obtain the important correspondence:

$\left(^{* *}\right) \min \left\|\hat{w}_{i}-U z\right\| R^{q}=\min \left\|\hat{w}_{i}-U z_{i}^{*}\right\| R^{q}$, while $\|z\| R^{m(m+3) / 2}>\left\|z_{i}^{*}\right\| R^{m(m+3) / 2}$.

Remark 2. Ratings $(*),\left({ }^{* *}\right)$ primarily depend on the "volume" of a posteriori information in the formation of matrix $U$ and vectors $\hat{w}_{i}$, namely if $q>m(m+3) / 2$, then it is likely to have the option (*), if $q \leq m(m+3) / 2$, it is likely that in the mathematical modeling of chemical-technological process there is position $\left({ }^{* *}\right)$.

\section{Optimization of the Mode of Chemical-Technological Process Based on Quadratic Interpolation of Its Functional Model}

An attractive idea to create engineering projects and algorithms that are adapted to changing conditions of studied (in the frameworks of these projects) chemical-technological processes, requires the use of nonlinear regression models of class (5) which are optimal flexible (tunable) during the variety of experimental data. Therefore the parametric identification of the functional model of chemical-technological process of class of regressions (5) studied in the previous section was necessary "technological" requirement in solving the problem of "synthesis control" $v \in R^{m}$. However, there are many variants of such control and it is necessary to choose among them the one that would be optimal from the viewpoint of some formal criteria which characterizes a certain physicaltechnical quality of this control. Below we will consider the problem of optimization in formulation (iii) (with a priority selection of coefficients $r_{i}, 1 \leq i \leq n$ [11]) and will discuss the algorithmic technique of constructing the mode of optimal control. But before we will consider this problem in the variant of optimization of separate variable of chemical-technological process-vector $w(\omega+v) \in R^{n}$.

Proposition 4. Let $D_{i}=\left(B_{i}+B_{i}^{\mathrm{T}}\right) \in M_{m, m}(R),(i=1, \cdots, n)$, where $B_{i}$-matrix of identified regression system (5) and $\operatorname{rank} D_{i}=m$. Then by varying the coordinates of vector of controlled actions $v \in R^{m}$ index of functional quality of chemical-technological process of form

$$
J_{i}(v)=w_{i}(\omega+v),(i=1, \cdots, n)
$$

may have an inner extremum (at $\varepsilon(\omega, v)=0$ ) only at the point (mode)

$$
v^{*}=-D_{i}^{-1} A^{\mathrm{T}} e_{i} \in R^{m}
$$

where $\left\{e_{1}, \cdots, e_{n}\right\}$-standard basis in $R^{n}$, and holds the positions:

-if $v^{\mathrm{T}} D_{i} v-$ negative definite quadratic form, then the functional of quality $J_{i}(v)$ has a maximum in the mode $v^{*}$;

-if $v^{\mathrm{T}} D_{i} v$-positive definite quadratic form, then the functional of quality $J_{i}(v)$ has a minimum in the mode $v^{*}$;

-if $v^{\mathrm{T}} D_{i} v$-quadratic form taking both positive and negative values, then the functional of quality $J_{i}(v)$ doesn't have maximum or minimum in the mode $v^{*}$.

Remark 3. In the first two cases of definite sign of quadratic form $v^{\mathrm{T}} D_{i} v$ extremal point (9)—point of elliptic type in the third case this point has a hyperbolic type (saddle point).

Proof of Proposition 4. Since

$$
J_{i}(v)=c_{i}+e_{i}^{\mathrm{T}} A v+v^{\mathrm{T}} B_{i} v
$$

then the necessary conditions of a local extremum have ([5] p. 500) the form:

$$
\begin{gathered}
\partial\left(c_{i}+e_{i}^{\mathrm{T}} A v+v^{\mathrm{T}} B_{i} v\right) / \partial v_{1}=0 \\
\vdots \\
\partial\left(c_{i}+e_{i}^{\mathrm{T}} A v+v^{\mathrm{T}} B_{i} v\right) / \partial v_{m}=0
\end{gathered}
$$


that is equivalent to the system of equations (below $\left\{\hat{e}_{1}, \cdots, \hat{e}_{m}\right\}$-standard basis in $R^{m}$ )

$$
\begin{gathered}
e_{i}^{\mathrm{T}} A \hat{e}_{1}+\hat{e}_{1}^{\mathrm{T}} B_{i} v+\hat{e}_{1}^{\mathrm{T}} B_{i}^{\mathrm{T}} v=0 \\
\vdots \\
e_{i}^{\mathrm{T}} A \hat{e}_{m}+\hat{e}_{m}^{\mathrm{T}} B_{i} v+\hat{e}_{m}^{\mathrm{T}} B_{i}^{\mathrm{T}} v=0
\end{gathered}
$$

which (as it has been easily seen) determine in the space $R^{m}$ geometric coordinates (9) for a stationary point of the functional $J_{i}(v)$.

On the other hand, the definite sign of the second differential

$$
d^{2} J_{i}\left(v^{*}\right)=\sum_{1 \leq g, p \leq m} \partial^{2} J_{i}(v) / \partial v_{g} v_{p} / v^{*} v_{g} v_{p}=v^{\mathrm{T}} D_{i} v
$$

determines sufficient conditions ([5] p. 503) for a local extremum at the critical point (9).

Coordinates of the stationary point (9) allow us to answer the question about the meaning of the functional $J_{i}(v)$, when this point is the point of a relative minimum (or maximum) which states the following sentence:

Corollary 2. If $D_{i}$-is a negative definite (similarly positive definite) matrix, then maximum (or minimum) value of the functional $J_{i}\left(v^{*}\right)$ equals

$$
J_{i}\left(v^{*}\right)=c_{i}-e_{i}^{\mathrm{T}} A D_{i}^{-1} A^{\mathrm{T}} e_{i} / 2
$$

where $c_{i}$-i-th coordinate of the vector $c \in R^{n}$ of system (5).

The proof is constructed by substituting (9) in (1).

Now we will turn to the study of more complex (task (iii)) variant of the problem of optimization of characteristics of chemical-technological process which plays the fundamental role in a more realistic and at the same time more difficult problems in calculating the optimal technological parameters of the mode of functioning of chemical-technological process. Its basis is the methodological position-each functional $J_{i}(v), 1 \leq i \leq n$ with appropriate interpretation can be generalized to the target functional (3). Thus, Proposition 4 and Formula (9) allow to find exact geometric coordinates of stationary point of the problem of optimization (3) for a finite sequence of algorithmic actions.

Proposition 4. Let $r=\operatorname{col}\left(r_{1}, \cdots, r_{n}\right), D_{i}=\left(B_{i}+B_{i}^{\mathrm{T}}\right) \in M_{m, m}(R), 1 \leq i \leq n$, where each $B_{i}$-matrix of regression system (5), $D(r)=\left(r_{1} D_{1}+\cdots+r_{n} D_{n}\right) \in M_{m, m}(R)$ and rank $D(r)=m$. Then the stationary point $v^{*} \in R^{m}$ of the problem of optimization (3) has the form

$$
v^{*}=-D(r)^{-1} A^{\mathrm{T}} r \in R^{m}
$$

while a sufficient condition that $v^{*}$ provides the quality for a chemical-technological process

$$
\begin{aligned}
& \max \left\{F\left(v: v \in R^{m}\right)\right\} \\
& F(v)=\sum_{i=1, \cdots, n} r_{i} J_{i}(v)
\end{aligned}
$$

is the following requirement: stationary point $v^{*}$ has an elliptic type which is equivalent to the position:

$$
\operatorname{det}\left[d_{i j}\right]_{p}<0, p=1, \cdots, m
$$

where $\left[d_{i j}\right]_{p} \in M_{p p}(R), p=1, \cdots, m$-principal submatrices ([7] p. 30) of matrix $D(r)$ whichis equivalent to: own values $\lambda_{p}$ of matrix Dcorrespond to inequalities

$$
\lambda_{p}<0, p=1, \cdots, m
$$

Proof. Main provisions of the proof repeat the conclusion of Proposition 4 that's why we are restricted by the scheme of the proof. Necessary conditions of a local extremum have ([5] p. 500) the form of $n$ equations:

$$
\begin{gathered}
r_{1} \partial\left(c_{1}+e_{1}^{\mathrm{T}} A v+v^{\mathrm{T}} B_{1} v\right) / \partial v_{1}+\cdots+r_{i} \partial\left(c_{i}+e_{i}^{\mathrm{T}} A v+v^{\mathrm{T}} B_{i} v\right) / \partial v_{1}+\cdots+r_{n} \partial\left(c_{n}+e_{n}^{\mathrm{T}} A v+v^{\mathrm{T}} B_{n} v\right) / \partial v_{1}=0, \\
\vdots \\
r_{1} \partial\left(c_{1}+e_{1}^{\mathrm{T}} A v+v^{\mathrm{T}} B_{1} v\right) / \partial v_{m}+\cdots+r_{i} \partial\left(c_{i}+e_{i}^{\mathrm{T}} A v+v^{\mathrm{T}} B_{i} v\right) / \partial v_{m}+\cdots+r_{n} \partial\left(c_{n}+e_{n}^{\mathrm{T}} A v+v^{\mathrm{T}} B_{n} v\right) / \partial v_{m}=0
\end{gathered}
$$


which is equivalent to the system of $n$ equations:

$$
\begin{gathered}
r_{1}\left(e_{1}^{\mathrm{T}} A \hat{e}_{1}+\hat{e}_{1}^{\mathrm{T}} B_{1} v+\hat{e}_{1}^{\mathrm{T}} B_{1}^{\mathrm{T}} v\right)+\cdots+r_{i}\left(e_{i}^{\mathrm{T}} A \hat{e}_{1}+\hat{e}_{1}^{\mathrm{T}} B_{i} v+\hat{e}_{1}^{\mathrm{T}} B_{i}^{\mathrm{T}} v\right)+\cdots+r_{n}\left(e_{n}^{\mathrm{T}} A \hat{e}_{1}+\hat{e}_{1}^{\mathrm{T}} B_{n} v+\hat{e}_{1}^{\mathrm{T}} B_{n}^{\mathrm{T}} v\right)=0 \\
\vdots \\
r_{1}\left(e_{1}^{\mathrm{T}} A \hat{e}_{m}+\hat{e}_{m}^{\mathrm{T}} B_{1} v+\hat{e}_{m}^{\mathrm{T}} B_{1}^{\mathrm{T}} v\right)+\cdots+r_{i}\left(e_{i}^{\mathrm{T}} A \hat{e}_{m}+\hat{e}_{m}^{\mathrm{T}} B_{i} v+\hat{e}_{m}^{\mathrm{T}} B_{i}^{\mathrm{T}} v\right)+\cdots+r_{n}\left(e_{n}^{\mathrm{T}} A \hat{e}_{m}+\hat{e}_{m}^{\mathrm{T}} B_{n} v+\hat{e}_{m}^{\mathrm{T}} B_{n}^{\mathrm{T}} v\right)=0
\end{gathered}
$$

the last system leads to the solution (10). $\square$

If algebraic conditions (11) (equivalent to (12)) don't meet then the critical point (10) of functional quality of chemical-technological process is either ([7] p. 288) hyperbolic (i.e. saddle point) or parabolic point and therefore it requires additional geometric analysis of critical controlled variables of chemical-technological process expressed by the formula (10).

Speaking more formally we can quote: the presence of a saddle point warrants a change in at least one (but not all) inequality “<” from (11) (or (12)) on inequality ">”; while a similar change of relation "<" on " $\leq$ " may causes the structure of parabolic point in the analytical solution of the problem of optimization.

The presented approach methodologically extends the standard procedure of planning experiment of chemical-technological process. Thus, if the calculated (predicted) coordinates of the stationary point (10) of any chemical-technological parameters are outside the area of adequacy of the identified model (5), it is necessary to conduct an additional experiment while implement chemical-technological process with vector $v \in R^{m}$, which is close to critical controlled variables of chemical-technological process (10), followed by the introduction of a given result in the extended (thus) matrix of experimental data $U$. Then it is necessary to recalculate [12] all above steps of the process of optimization of controlled variables of chemical-technological process; if necessary this additional experiment, parametric identification of chemical-technological process of the form (2) and quadratic optimization (3) should be repeated.

\section{Numerical Modeling of the Mode of Chemical-Technological Process}

The previous sections have been conceived as an attempt to bring "compactly" together under the same terms and notations large but diverse enough number of rigorous mathematical results that are dedicated to such a broad topic as multivariate regression analysis with emphasis on methods of covariant-tensor representation of functional derivatives (Frechet derivatives) involving the method of the least squares and their practical application to the optimization of complex multivariate processes. Next section is devoted to a detailed study of related concepts while the basic attention is focused on experimental testing of the theoretical results from Paragraphs 1 - 4 on the basis of experimental studies of process of low-temperature sulfur-chromium.

Numerical modeling was carried out in the environment of software package [12] which solves practical issues of regression-tensor modeling of multivariate chemical-technological process. Rigorous analytical interpretations of multiple technical constraints are determined on its base, imposing as complex nonlinear constraints both theoretical and empirical character and ensuring the adequacy of investigated model by a posteriori data - the problem of identification by the method of the least squares of coordinates of covariant tensors (both linear and bilinear) of multidimensional nonlinear regression. At the final step this modeling "connects" algorithms of quadratic optimization of synthesis of optimal technological characteristics of behavior of chemicaltechnological process.

Without loss of generality as a reference mode of process of low-temperature sulfur-chromium we can take some point $\omega$ of space $R^{m}$ empirically selected from the overall composition of the experimental data; it is clear that in this case coordinates $v_{1}, \cdots, v_{m}$ of vector $v$ should be regarded as deviations with respect to the designed mode $\omega \in R^{m}$ :

Parameters of reference mode: $\omega_{1}=125^{\circ} \mathrm{C}, \omega_{2}=0.92$ hour, $\omega_{3}=43 \% \mathrm{NaOH}, \omega_{4}=0.5 \% \mathrm{~S}, \omega_{5}=1 \% \mathrm{Na}_{2} \mathrm{~S}, \omega_{6}$ $=2 \% \mathrm{Na}_{2} \mathrm{~S}_{2} \mathrm{O}_{3}, \omega_{7}=10 \% \mathrm{CrO}_{3}$.

Multivariate synthesis of sulfur-chromium layer in the series from field experiments $q$ (due to $m=7$ and p. $(* *)$ of Corollary 1 the number of experiments $q \leq 35$ ) we will describe with the following chemical-technological variables:

Input data: $v=\operatorname{col}\left(v_{1}, v_{2}, v_{3}, v_{4}, v_{5}, v_{6}, v_{7}\right) \in R^{7}$-vector of variations with respect to the reference mode $\omega=\operatorname{col}\left(\omega_{1}, \omega_{2}, \omega_{3}, \omega_{4}, \omega_{5}, \omega_{6}, \omega_{7}\right)$ of controlled features of process of low-temperature sulfur-chromium: 
$v_{1}$-variation (to $\omega_{1}$ ) of temperature of process $10^{-2}\left[{ }^{\circ} \mathrm{C}\right]$,

$v_{2}$-variation (to $\omega_{2}$ ) of duration of process [hour],

$v_{3}$-variation (to $\omega_{3}$ ) of concentration of hydroxide of sodium $10^{-2} \mathrm{NaOH}$ [\%],

$v_{4}$-variation (to $\omega_{4}$ ) of concentration of sulfur S [\%],

$v_{5}$-variation (to $\omega_{5}$ ) of concentration of sulfide of sodium $\mathrm{Na}_{2} \mathrm{~S}$ [\%],

$v_{6}$-variation (to $\omega_{6}$ ) of concentration of hypophosphite of sodium $10^{-1} \mathrm{Na}_{2} \mathrm{~S}_{2} \mathrm{O}_{3}$ [\%],

$v_{7}$-variation (to $\omega_{7}$ ) of concentration of three oxide of chromium $10^{-1} \mathrm{CrO}_{3}$ [\%].

Output data: $\quad w=w_{1} \in R$-characteristicof sulfur-chromium layerof plunger assembly:

$w_{1}$-thickness of sulfur-chromium layer [micro-métre].

The solution of the problem of parametric identification (2) for the regression Equation (5) of process of lowtemperature sulfur-chromium presented in Table 1 by Proposition 3 and calculations in the software environment [12], has the form:

$$
\begin{aligned}
w_{1}(\omega+v)= & 9-82.2838 v_{1}-40.3136 v_{2}+2.4814 v_{3}+24.8137 v_{4}+24.8137 v_{5}+12.4069 v_{6}+24.8137 v_{7} \\
& -186.0956 v_{1}^{2}-180.2625 v_{1} v_{2}-12.3797 v_{1} v_{3}-123.7968 v_{1} v_{4}-123.7968 v_{1} v_{5}-61.8984 v_{1} v_{6} \\
& -123.7968 v_{1} v_{7}-62.7154 v_{2}^{2}+15.1415 v_{2} v_{3}+151.4146 v_{2} v_{4}+151.4146 v_{2} v_{5}+75.7073 v_{2} v_{6} \\
& +151.4146 v_{2} v_{7}-0.0867 v_{3}^{2}-0.8668 v_{3} v_{4}-0.8668 v_{3} v_{5}-0.4334 v_{3} v_{6}-0.8668 v_{3} v_{7} \\
& -8.6681 v_{4}^{2}-8.6681 v_{4} v_{5}-4.334 v_{4} v_{6}-8.6681 v_{4} v_{7}-8.6681 v_{5}^{2} \\
& -4.334 v_{5} v_{6}-8.6681 v_{5} v_{7}-2.167 v_{6}^{2}-4.334 v_{6} v_{7}-8.6681 v_{7}^{2}
\end{aligned}
$$

Critical analysis of efficiency of model of the mathematical description of process of low-temperature sulfurchromium expressed by Equations (13) gives the comparison of the last two columns of Table 1; here $w_{1}$ - experiment, $\hat{w}_{1}$-forecast according to (13). The graphic illustration of the index of quality $J_{1}(v)=w_{1}(\omega+v)-w_{1}(\omega)$ at varying temperature, duration of sulfur-chromium and concentrations of solution depending on the scale (see Table 1) variations with respect to the mode $\omega$ is shown in Figures 1-9.

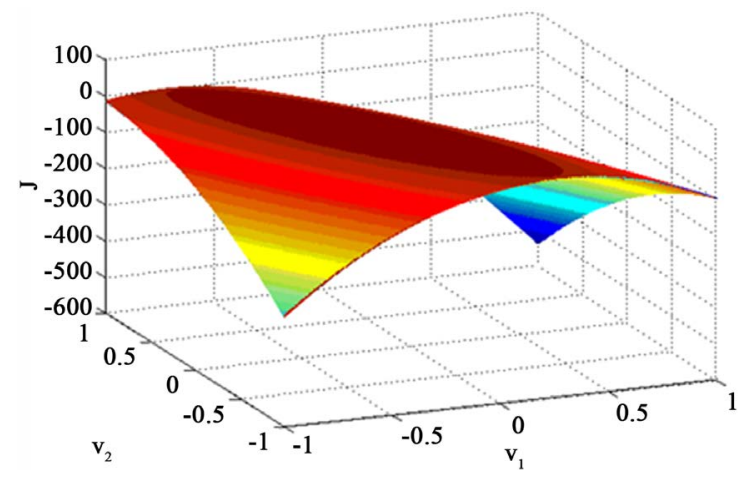

Figure 1. Dependence of thickness of sulfur-chromium layer from the temperature and duration of process of plunger assembly- $J_{1}\left(v_{1}, v_{2}\right)$.

Table 1. Process of low-temperature of sulfur-chromium.

\begin{tabular}{ccccccccccc}
\hline Number of experiment & \multicolumn{9}{c}{ Parameters of mode of coating of sulfur-chromium layer } & \multicolumn{2}{c}{ Thickness of layer } \\
\hline № & $\omega_{1}+v_{1}$ & $\omega_{2}+v_{2}$ & $\omega_{3}+v_{3}$ & $\omega_{4}+v_{4}$ & $\omega_{5}+v_{5}$ & $\omega_{6}+v_{6}$ & $\omega_{7}+v_{7}$ & $w_{1}(\omega+v)$ & $\hat{w}_{1}(\omega+v)$ \\
1 & 1 & 0.5 & 0.4 & 0.2 & 0.7 & 0.05 & 0.7 & 7.2 & 7.2 \\
2 & 1.1 & 0.7 & 0.41 & 0.3 & 0.8 & 0.1 & 0.8 & 8.1 & 8.1 \\
3 & 1.2 & 0.83 & 0.42 & 0.4 & 0.9 & 0.15 & 0.9 & 8.7 & 8.7 \\
$4-\omega$ & 1.25 & 0.92 & 0.43 & 0.5 & 1 & 0.2 & 1 & 9 & 9 \\
5 & 1.3 & 1 & 0.44 & 0.6 & 1.1 & 0.25 & 1.1 & 9.5 & 9.5 \\
6 & 1.35 & 1.8 & 0.45 & 0.7 & 1.2 & 0.3 & 1.2 & 9.5 & 9.5 \\
7 & 1.4 & 1.17 & 0.46 & 0.8 & 1.3 & 0.35 & 1.3 & 9.5 & 9.5 \\
\hline
\end{tabular}




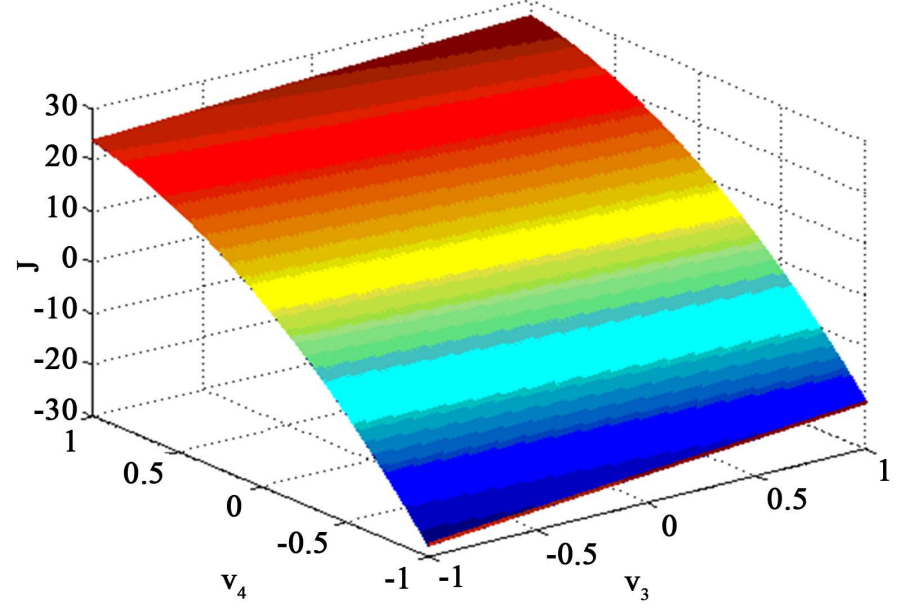

Figure 2. Dependence of thickness of sulfur-chromium layer from concentrations of hydroxide of sodium and sulfur- $J_{1}\left(v_{3}, v_{4}\right)$.

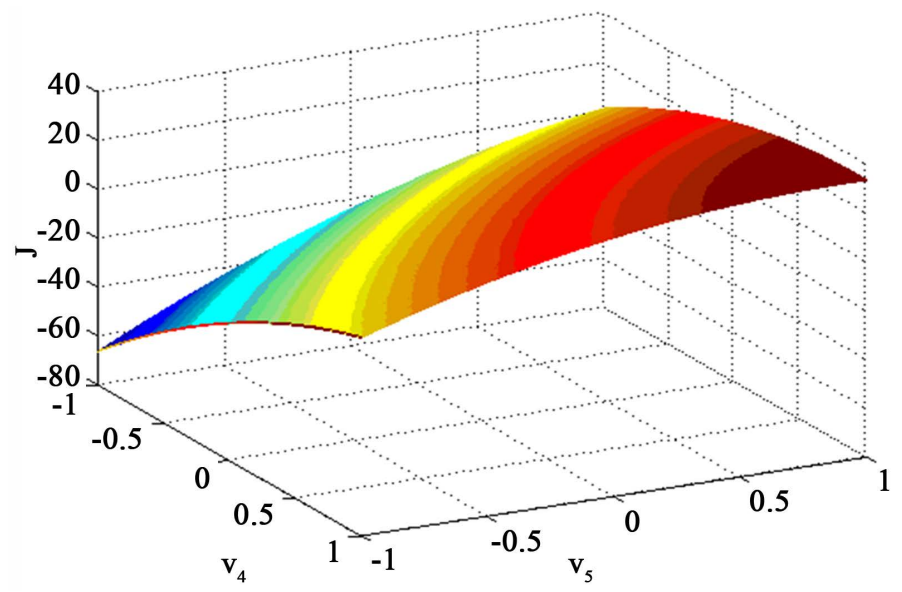

Figure 3. Dependence of thickness of sulfur-chromium layer from concentrations of sulfur and sulfide of sodium- $J_{1}\left(v_{4}, v_{5}\right)$.

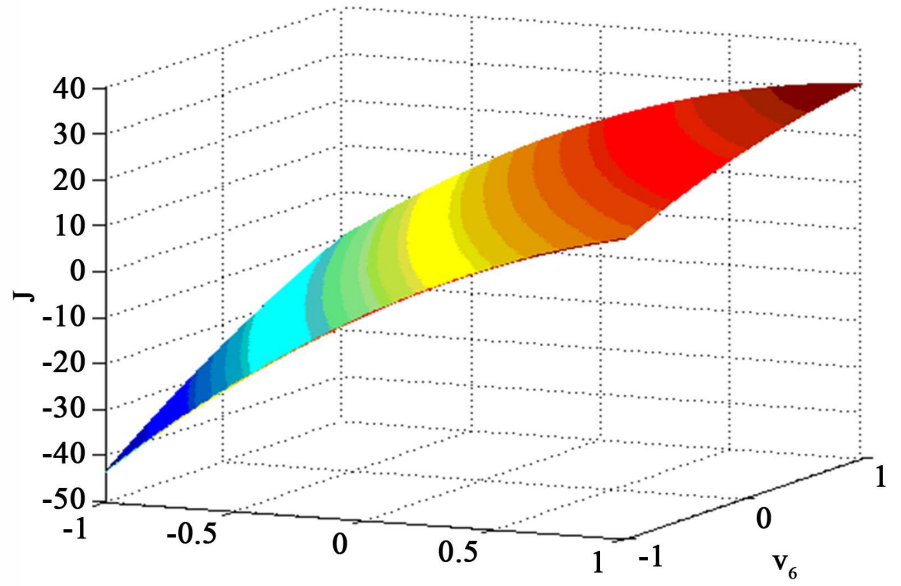

Figure 4. Dependence of thickness of sulfur-chromium layer from concentrations of sulfide of sodium and hypsosulfide of sodium- $J_{1}\left(v_{5}, v_{6}\right)$. 


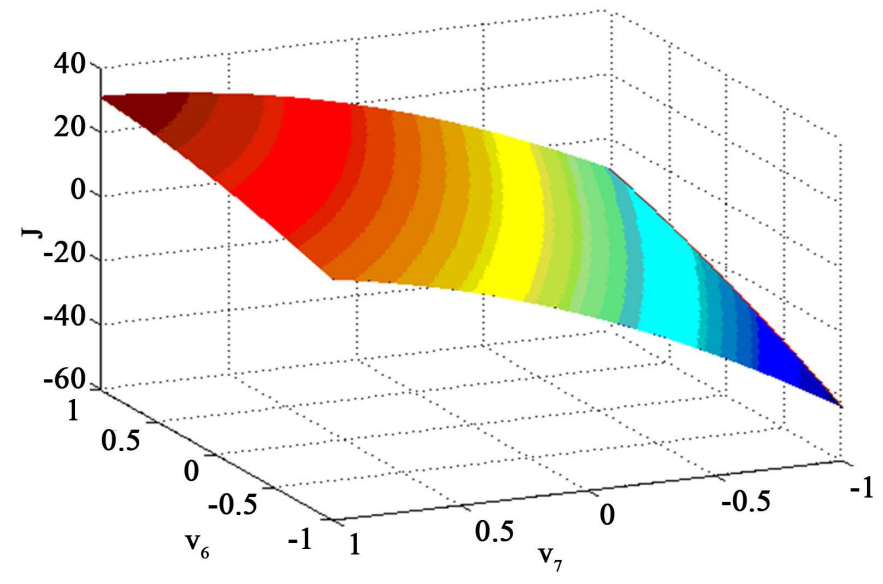

Figure 5. Dependence of thickness of sulfur-chromium layer from concentrations of hypophosphite of sodium and chromium oxide- $J_{1}\left(v_{6}, v_{7}\right)$.

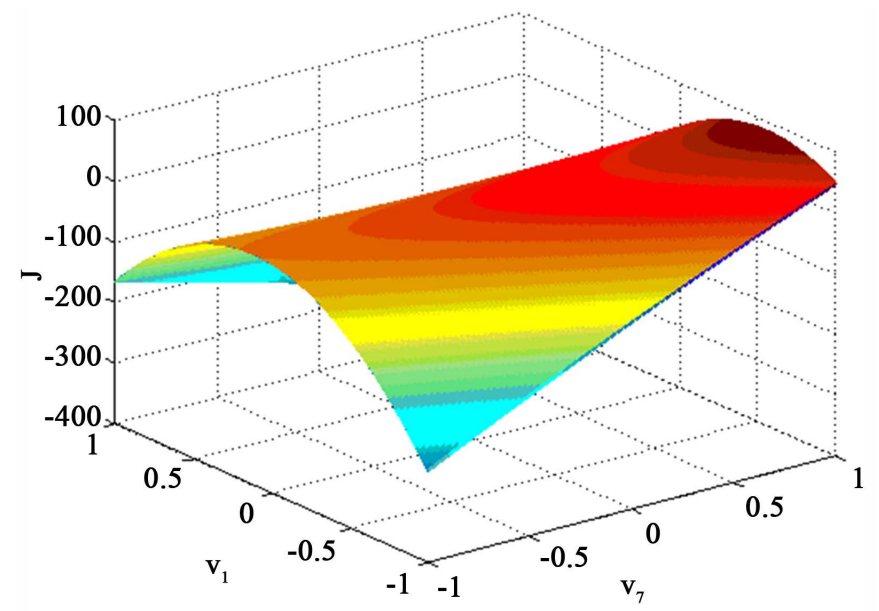

Figure 6. Dependence of thickness of sulfur-chromium layer from concentration of chromium oxide and temperature- $J_{1}\left(v_{7}, v_{1}\right)$.

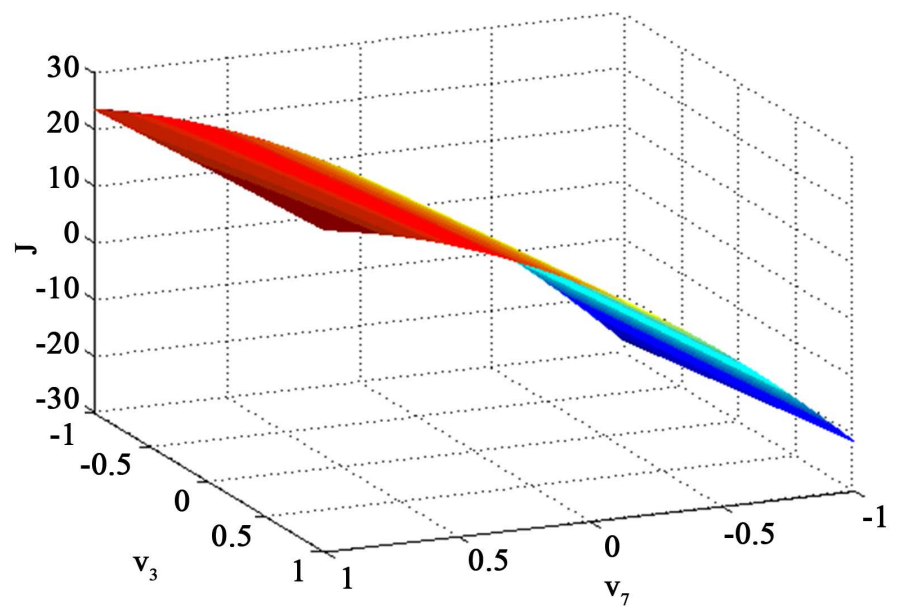

Figure 7. Dependence of thickness of sulfur-chromium layer from concentrations of chromium oxide and hydroxide of sodium $-J_{1}\left(v_{7}, v_{3}\right)$. 


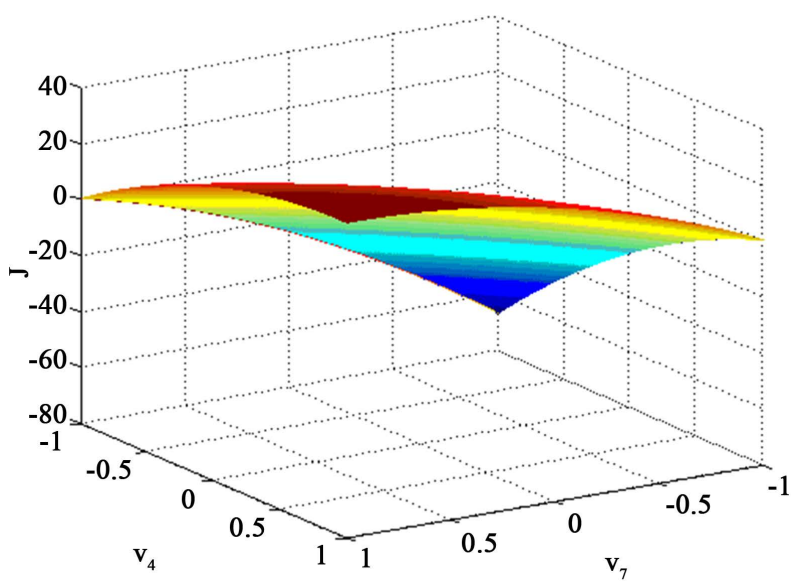

Figure 8. Dependence of thickness of sulfur-chromium layer from concentrations of chromium oxide and sulfur- $J_{1}\left(v_{7}, v_{4}\right)$.

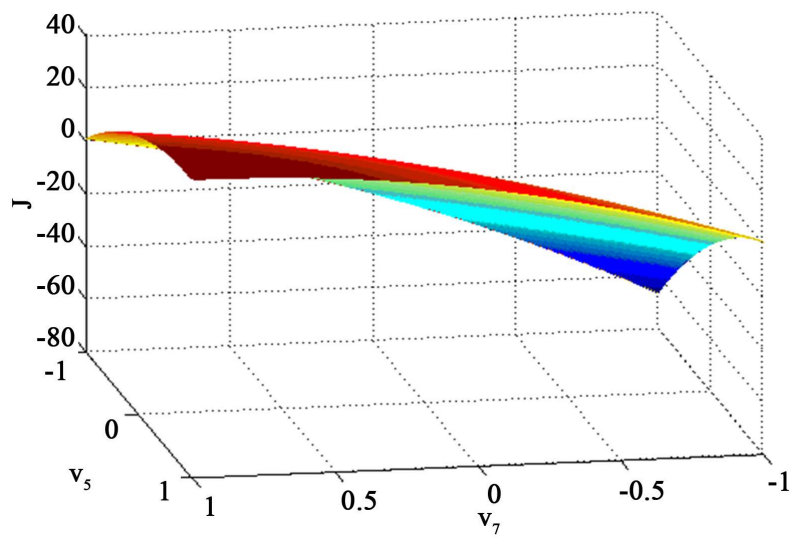

Figure 9. Dependence of thickness of sulfur-chromium layer from concentrations of chromium oxide and sulfide of sodium $-J_{1}\left(v_{7}, v_{5}\right)$.

\section{Optimization of Mode of Chemical-Technological Process}

Combining the results of Paragraphs 1 - 4 sulfur-chromium mode providing maximum thickness of physical structure of sulfur-chromium layer of machined surface of precision item we will contact with the solution of the optimization problem of the following form:

$$
\max \left\{F(v): v \in R^{7}\right\}, F(v):=J_{i}(v)
$$

Development of new technological methods of processing of metals requires an adequate mathematical model allowing to predict interrelated effect of various factors of physical-chemical environment of the metalworking and mechanical-geometric characteristics of the treated surface of the item on the obtained results. Mathematical model of optimization (14) for a multivariate process of sulfur-chromium gives such an opportunity, namely, to identify the most critical parameters and set determined areas of improvement of used and developed technological plants for obtaining sulfur-chromium layer. Proposition 4 and Formula (9) allowing to calculate geometric coordinates of the stationary point for the problem of optimization determine (in terms of system (13)) the following highly effective technological parameters of the mode of sulfur-chromium: by virtue of system (5) (or that is equivalent to the Equations (13)) a stationary point (9) in the coordinate representation (of a row-vector) has the form:

$$
\omega^{\mathrm{T}}+v^{* \mathrm{~T}}=\left[\begin{array}{lllllll}
1.1593 & 0.6791 & 0.3475 & 0.4917 & 0.9917 & 0.1835 & 0.9917
\end{array}\right]
$$


or the same in the physical dimensions of given "reference" from the mode $\omega$ :

$$
\omega^{\mathrm{T}}+v^{* \mathrm{~T}}=\left[\begin{array}{llllll}
115.93^{\circ} \mathrm{C} & 0.6791 \text { hour } \quad 34.75 \% & 0.4917 \% & 0.9917 \% & 1.835 \% & 9.917 \%
\end{array}\right]
$$

Mathematical result obtained above (the coordinates of the stationary point of sulfur-chromium mode (9)) is in accordance with the logic of physical reasoning; illustration $F_{1}(v)=w_{1}\left(\omega+v^{*}+v\right)-w_{1}\left(\omega+v^{*}\right)$ see on the Figures 10-18.

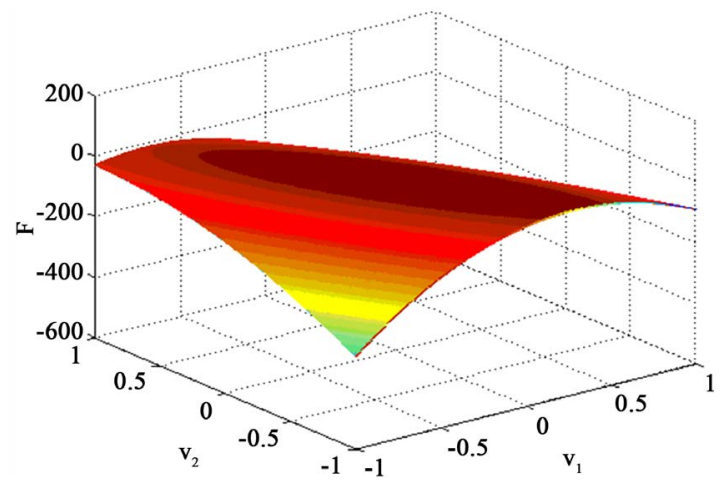

Figure 10. Detail of the target functional $F\left(v_{1}, v_{2}\right)$ in the vicinity of the stationary point (15).

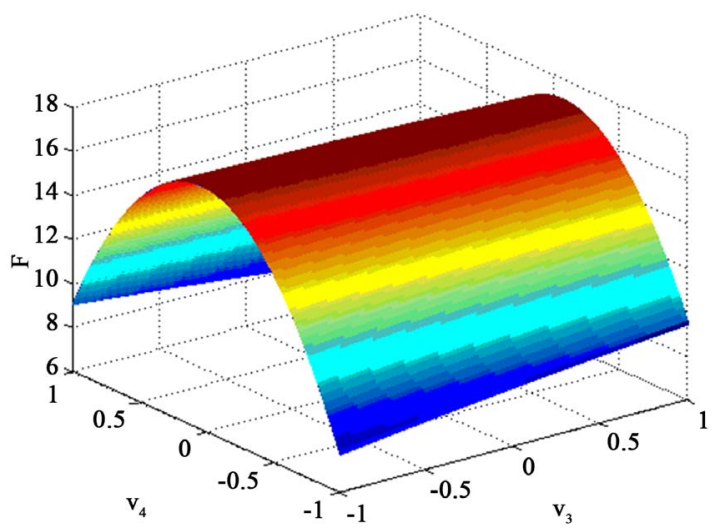

Figure 11. Detail of the target functional $F\left(v_{3}, v_{4}\right)$ in the vicinity of the stationary point (15).

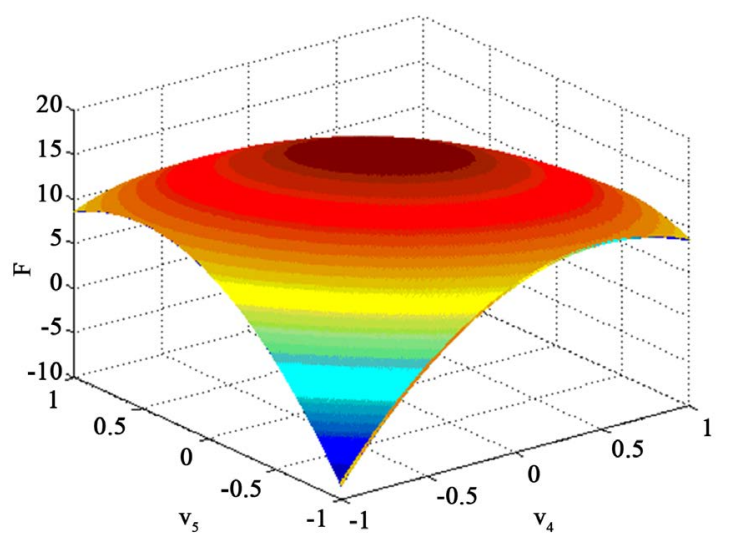

Figure 12. Detail of the target functional $F\left(v_{4}, v_{5}\right)$ in the vicinity of the stationary point (15). 


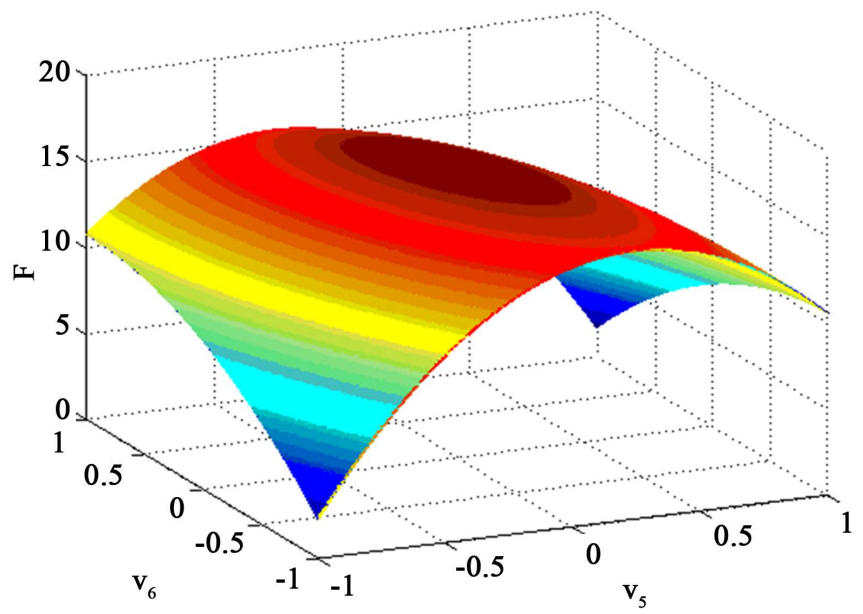

Figure 13. Detail of the target functional $F\left(v_{5}, v_{6}\right)$ in the vicinity of the stationary point (15).

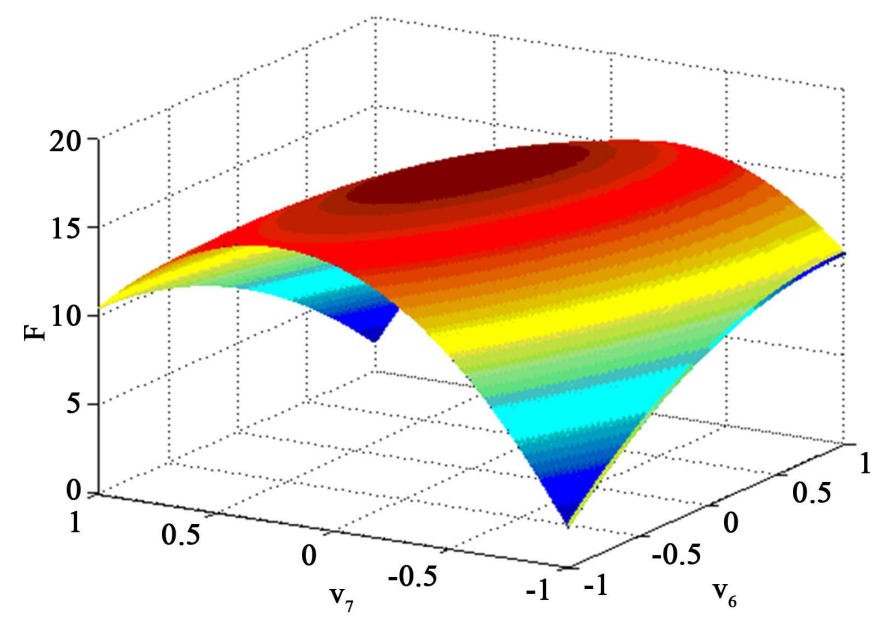

Figure 14. Detail of the target functional $F\left(v_{6}, v_{7}\right)$ in the vicinity of the stationary point (15).

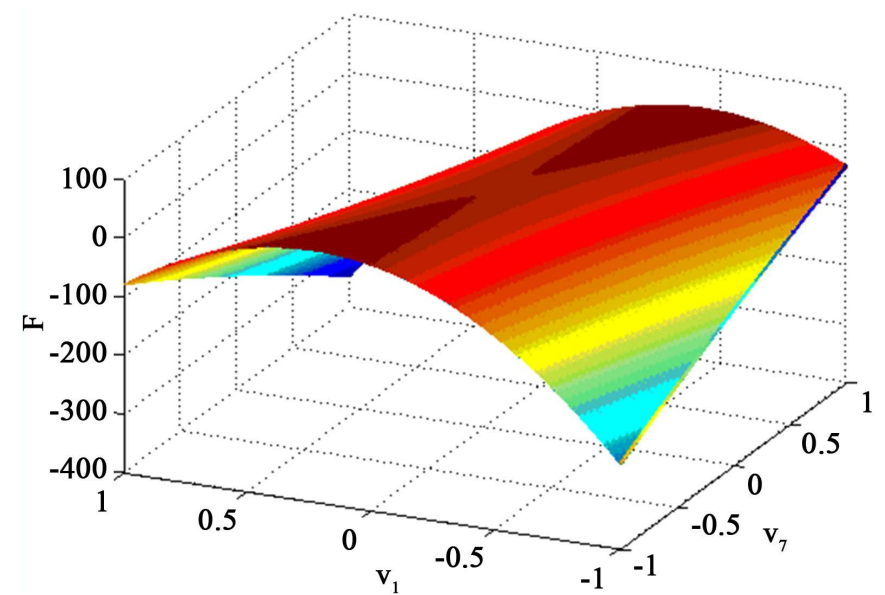

Figure 15. Detail of the target functional $F\left(v_{7}, v_{1}\right)$ in the vicinity of the stationary point (15). 


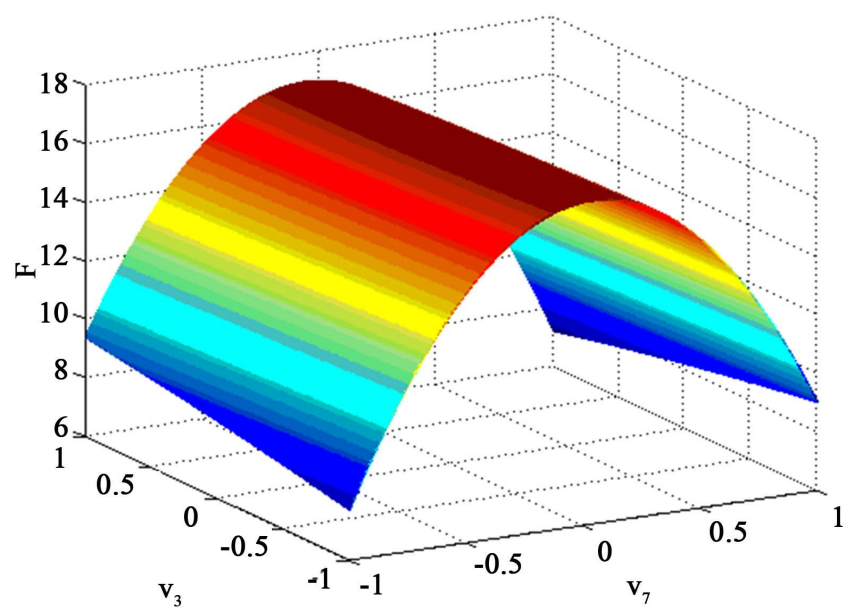

Figure 16. Detail of the target functional $F\left(v_{7}, v_{3}\right)$ in the vicinity of the stationary point (15).

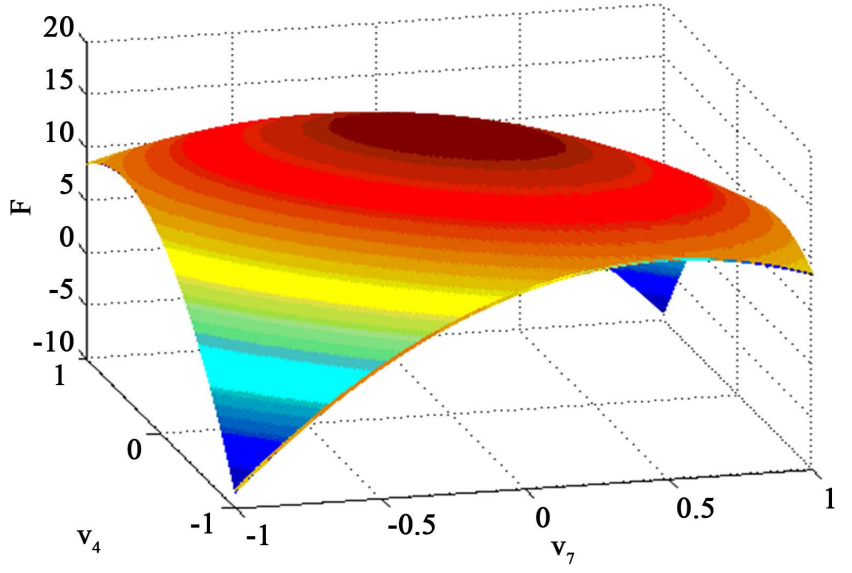

Figure 17. Detail of the target functional $F\left(v_{7}, v_{4}\right)$ in the vicinity of the stationary point (15).

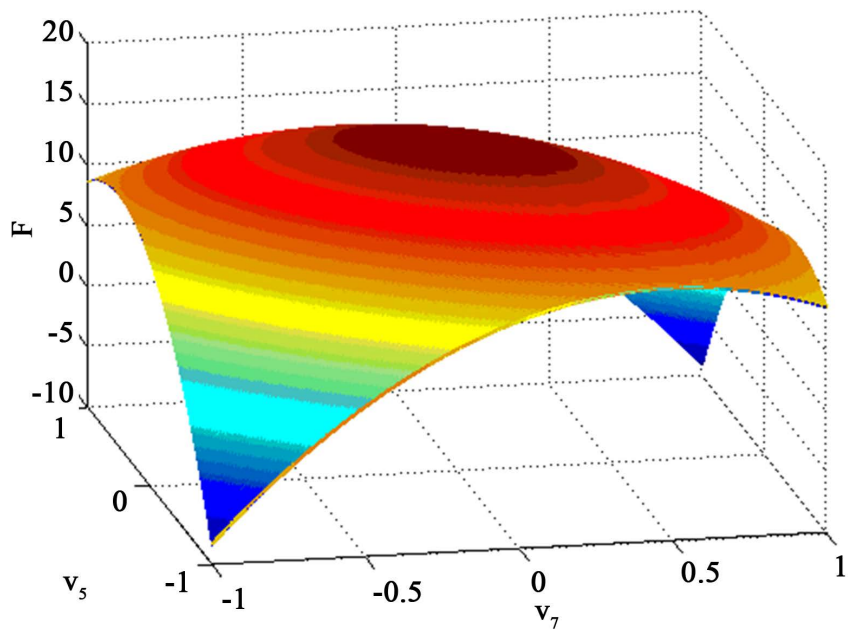

Figure 18. Detail of the target functional $F\left(v_{7}, v_{5}\right)$ in the vicinity of the stationary point (15). 
Since own values of the matrix $D_{1}$ respectively equal

$$
\begin{aligned}
& \lambda_{1}=-246.1546, \lambda_{2}=-178.7824, \lambda_{3}=-4.3340, \lambda_{4}=-4.3340, \\
& \lambda_{5}=-1.3357, \lambda_{6}=-0.0540, \lambda_{7}=157.9257
\end{aligned}
$$

then it speaks about the stationary saddle point of the functional $F(v)$.

According to (12) and (16) in the obtained stationary point $v^{*}$ functional $F(v)$ reaches its "max" in the variables $v_{1}, \cdots, v_{6}$ and respectively its "min" in $v_{7}$.

The foregoing discussion can be summarized in one sentence: if there is $\omega_{7}=9.917 \% \mathrm{CrO}_{3}$, then it is necessary to fulfill the conditions

$$
\begin{aligned}
& \omega_{1}=115.93^{\circ} \mathrm{C}, \omega_{2}=0.6791 \text { hour, } \omega_{3}=34.75 \% \mathrm{NaOH}, \\
& \omega_{4}=0.4917 \% \mathrm{~S}, \omega_{5}=0.9917 \% \mathrm{Na}_{2} \mathrm{~S}, \omega_{6}=1.835 \% \mathrm{Na}_{2} \mathrm{~S}_{2} \mathrm{O}_{3}
\end{aligned}
$$

if the position $\omega_{7}=\beta \neq 9.917 \% \mathrm{CrO}$ is implemented, then it is necessary to decide the correction of the problem (3), (14) of the form in full capacity including the identification (2)

$$
\max \left\{F\left(v, \omega_{7}\right): v \in R^{6}, \omega_{7}=\beta\right\}
$$

This rule is of course largely engineering (not mathematical); from a purely mathematical point of view it only specifies the behavior of chemical-technological process stating that in any case it is necessary to describe (to explain in a heuristic level) the original choice of the percentage in the solution of three chromium oxide $\mathrm{CrO}_{3}$. In this connection we will mention another unexpected result: the first six parameters $\omega_{i}, i=1, \cdots, 6$ in the optimal mode of chemical-technological process essentially depend on the seventh factor $\omega_{7}$-content of three chromium oxide.

\section{Conclusions}

The problem of the analytical description of the a posteriori set of data occurs in many sections of science and technology associated with the modeling and/or identification of cognitive systems. In this context the article discusses theoretical issues of regression-tensor modeling of multivariate chemical-technological process in the class of systems (1) and on its basis rigorous analytical interpretations are given which were imposed as nonlinear constraints of theoretical nature as providing the optimal mode of chemical-technological process.

In Paragraph 2a detailed mathematical study of the question of existence of regression model while particular attention was paid to the role of differential calculation (in the constructions of strong Frechet derivatives) in finite-dimensional Euclidean spaces for receiving qualitative conditions (Proposition 2) in the solution of the task of "satisfactory" modeling. In this regard we will note that the description of chemical-technological process by regression system (1) and differential models [13] is adequate considering assertion ([5] p. 495) about differential dependence of the solution of differential equation from initial-boundary conditions and parameters as well as Proposition 2.

The problem of identification of the method of least squares of coordinates of covariant tensors of both linear and bilinear is considered in Paragraph 3 of common positions formalized by criterion (2). In the large extent in this part of work the confirmation of algorithmic theory of nonlinear regression-tensor modeling of chemicaltechnological process in terms of designing rules for calculating parameters (8) by conditions suitable for the application of the optimal estimation (2) of operators of the regression model (1) was received in terms of Proposition 3.

In Paragraph 4 the importance of the theory of a posteriori mathematical modeling of chemical-technological process outlined in previous sections is confirmed by the fact that it is not only analytical (which is important itself) but the fact that it leads to efficient algorithms for synthesis of the optimal chemical-technological process. In this context the formula (9) for calculating geometric coordinates of the stationary point of the optimal mode of chemical-technological process was obtained according to the target criterion (3) as well as sufficient conditions are given to guarantee maximum quality of chemical-technological process in practice.

Paragraphs 5, 6 show the results of the numerical solution based on the experimental data of the problem of identification of bilinear tensors of nonlinear regression model of sulfur-chromium coating layer having the optimal thickness of sulfur-chromium layer. The stages of the numerical solution of the problem of parametric identification were considered while the detected deviations of calculated (predicted) values of synthesized sul- 
fur-chromium layer and experimental data aren't of fundamental nature in consequence of which effective mathematical method (finite chain of algebraic operations (9)) of calculating the optimal coating thickness providing the parameters of nonlinear multivariate mode of sulfur-chromium space of precision item was investigated and confirmed.

For a more complex chemical-technological process, a broader "dictionary of modeling" and the best knowledge of the theory of multivariate regression-tensor modeling are necessary to describe the structure of the functional (3) and use its properties due to additional research (in the spirit of [14]) of factors of nonlinearity of models (1):

- on identification and algorithmization of procedure of selection of weighted coefficients $r_{i}, 1 \leq i \leq n$ in (3) based on the implementation of algebraic conditions (11) and (12) providing elliptical nature of the stationary point (10);

- on the expansion of the linear-quadratic form of equations of regression (5) of "Taylor decomposition" of vector-function of regression of higher order;

- on registration of additional parameters-coordinates of vector-function of regression model such physicalmathematical parameters of chemical-technological process as surface hardness, wear resistance, coefficient of dry friction of treated surface as well as fragility of the resulting metal plating;

- on the development of nano-metric indicators of chemical-technological process and their qualitative account in the structure of nonlinear-tensor multidimensional regression model (1).

\section{Acknowledgements}

This work was supported by the Program “Leading Scientific Schools” (project no. NSh-5007.2014.09).

\section{References}

[1] Adler, Y.P., Markova, E.V. and Granovsky, Y.V. (1976) Planning of Experiment in Finding the Optimal Conditions. Nauka, Moscow, 255 p. (In Russian)

[2] Draper, N.R. and Smith, H. (1998) Applied Regression Analysis. John Wiley \& Sons Ltd., New York. [(2007) Vilyms, Moscow, 912 p. (In Russian)] http://dx.doi.org/10.1002/9781118625590

[3] Andrievskiy, B.R. and Fradkov, A.L. (2001) Elements of Mathematical Modeling in Software Environments MATLAB and SCILAB. Nauka, Saint-Petersburg, 288 p. (In Russian)

[4] Akivis, M.A. and Goldberg, V.V. (1972) Tensor Calculation. Nauka, Moscow, 352 p. (In Russian)

[5] Kolmogorov, A.N. and Fomin, S.V. (1976) Elements of the Theory of Functions and Functional Analysis. Nauka, Moscow, 544 p. (In Russian)

[6] Rusanov, V.A., Agafonov, S.V., Daneev, A.V. and Lyamin, S.V. (2013) Multivariable Regression Analysis of Optimal Conditions for a Physics-Chemical Process. International Journal of Materials Engineering and Technology, 10, 19-44.

[7] Horn, P. and Johnson, C. (1989) Matrix Analysis. Mir, Moscow, 656 p. (In Russian)

[8] Banach, S. (1972) Differential and Integral Calculus. Nauka, Moscow, 424 p. (In Russian)

[9] Gantmacher, F.R. (1988) Theory of Matrices. Nauka, Moscow, 552 p. (In Russian)

[10] Lankaster, P. (1982) Theory of Matrices. Nauka, Moscow, 272 p. (In Russian)

[11] Makarova, I.M., Vinogradskaya, T.M., Rubchinskiy, A.A. and Sokolova, V.B. (1982) Theory of Choice and Decision-Making. Nauka, Moscow, 328 p. (In Russian)

[12] Agafonov, S.V., Sharpinskiy, D.Y., Rusanov, V.A. and Udilov, T.V. (2008) Hybrid Regression Complex “GREEK”. Certificate of the Federal Service for Intellectual Property, Patents and Trademarks of the Registration of a Computer Program, № 2008614737.

[13] Kärger, J., Grinberg, F. and Heitjans, P. (2005) Diffusion Fundamentals. Leipziger Univ., Leipzig, 615 p.

[14] Ross, G.J. (1990) Nonlinear Estimation. Springer-Verlag, New York, 237 p. http://dx.doi.org/10.1007/978-1-4612-3412-8 
Scientific Research Publishing (SCIRP) is one of the largest Open Access journal publishers. It is currently publishing more than 200 open access, online, peer-reviewed journals covering a wide range of academic disciplines. SCIRP serves the worldwide academic communities and contributes to the progress and application of science with its publication.

Other selected journals from SCIRP are listed as below. Submit your manuscript to us via either submit@scirp.org or Online Submission Portal.
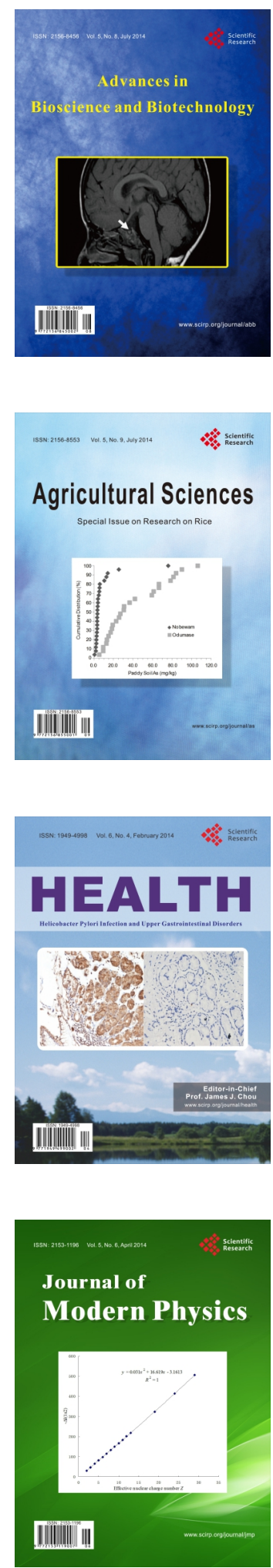
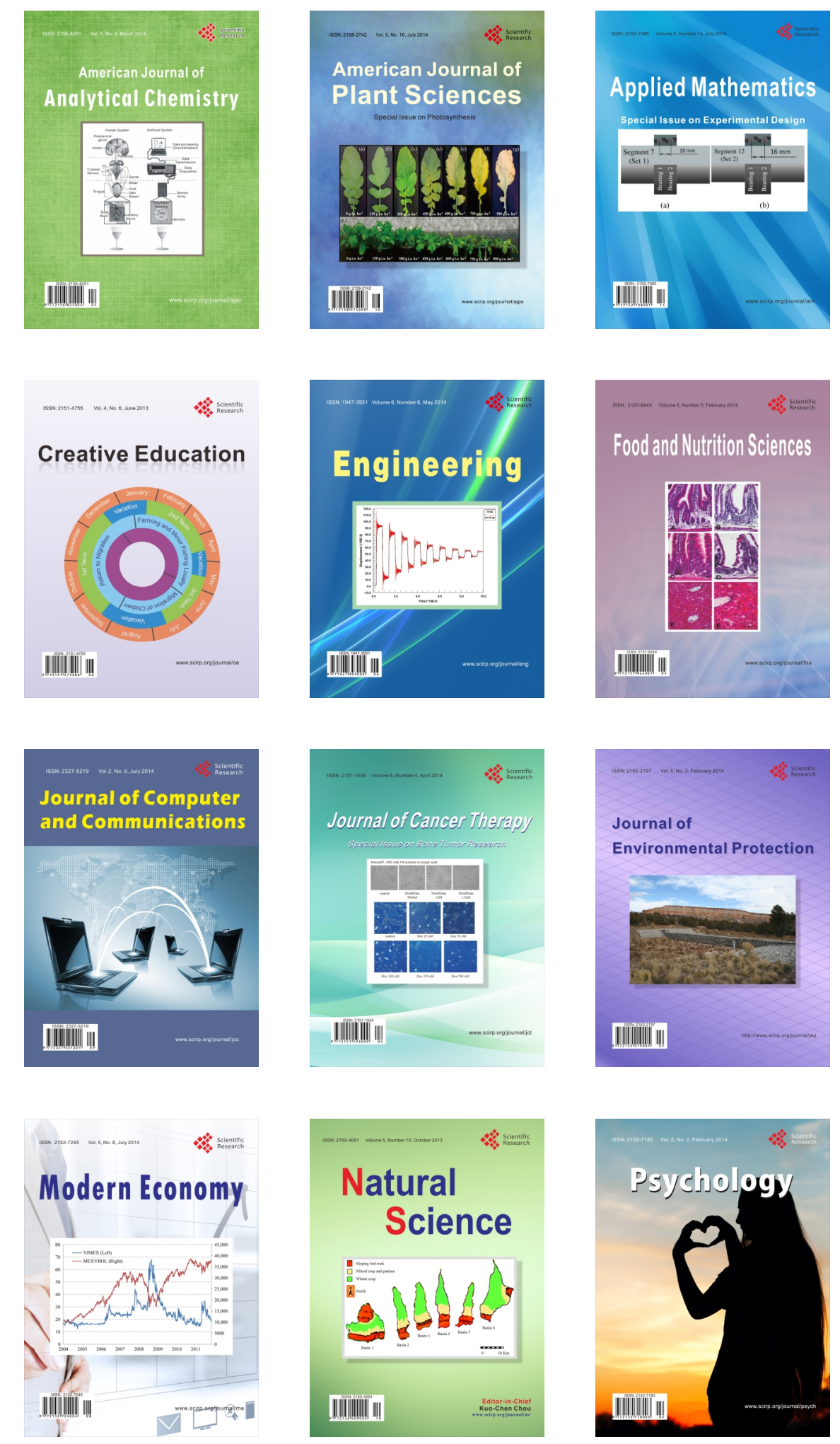